Reference Laboratory (now at the Public Health Laboratory, Portsmouth) received for identification 10 cultures of $\mathrm{Br}$. melitensis that had been isolated in the United Kingdom. One of these cultures was biotype 2 , and this was isolated on blood culture from an Italian aged 21 who had been resident in this country for two years; the source of infection was not discovered.

We wish to acknowledge the assistance in the clinical investigation of the following: Dr. D. K. Banerjee, Dr. P. D. Shanahan, Dr. J. C. Turner, general practitioners, and Dr. L. M. Webber, formerly medical registrar, Queen Mary's Hospital. Mr. A. J. Stevens, formerly of the Veterinary Investigation Laboratory, Leeds, kindly examined samples of the cheese.

\section{REFERENCES}

Cariello, M., and Tursi, L. (1964). Ann. Sanità pubbl., 25, 1041. Dalrymple-C̈hampneys, W. (1960). Brucella Infection and Undulant Fever in Man. London.

Davis, J. G. (1965). Cheese, vol. 1. London.
Gargani, G. (1952). Clin. vet. (Milano), 75, 257.

Gargani, G. (1952). Clin. vet. Milman, H. L., and Marquardt, J. C. (1951). f. Milk Fd Technol., 14, Iman,

Kerr, W. R., et al. (1968). f. med. Microbiol., 1, 181.

\title{
Controlled Trial of Dipyridamole in Cerebral Vascular Disease
}

\author{
JOAN ACHESON,* M.B., CH.B.; G. DANTA, $\dagger$ M.R.C.P., M.R.A.C.P. ; E. C. HUTCHINSON, $\ddagger$ M.D., F.R.C.P.
}

\begin{abstract}
Summary : A controlled double-blind study of the effect of dipyridamole was performed in 169 patients with established cerebral vascular disease. A dose of $400 \mathrm{mg}$. was used initially, given daily for an average of 14 months; the dose was then increased to $800 \mathrm{mg}$. daily for a further average period of 11 months. When the incidence of cerebral ischaemic episodes during treatment was compared in the drug-treated and placebo-treated groups no significant difference was found.
\end{abstract}

\section{Introduction}

Since the synthesis by Fischer and Roch (1951) of a new group of compounds containing two pyrimidine rings, there have appeared in the literature descriptions of the pharmacological properties and clinical effects of one of the derivatives, dipyridamole (Persantin). Experimentally this substance increases coronary blood flow and intercoronary anastomoses in dogs with surgically induced coronary artery constriction (Asada et al., 1962 ; Vineberg et al., 1962 ; Chiba, 1963 ; Fam et al., 1964; Meesmann and Bachmann, 1966); it increases the concentration of adenosine triphosphate in heart muscle (Laudahn, 1961 ; Siess, 1962 ; DeGraff and Lyon, 1963), and a recent experimental study suggested that it enhances the vasodilatory effect of adenosine triphosphate (Afonso and O'Brien, 1967). Adenosine-diphosphate-induced platelet aggregation is inhibited by dipyridamole both in vitro and in vivo (Emmons et al., 1965a, 1965b), and Sullivan et al. (1968b) showed that dipyridamole decreases platelet adhesiveness in patients with coronary artery disease.

Five double-blind studies in ischaemic heart disease have failed to show any beneficial effect of dipyridamole (Foulds and Mackinnon, 1960 ; Zion and Bradlow, 1961 ; Soloff et al., 1962 ; Newhouse and McGregor, 1965 ; Sbar and Schlant, 1967). In one double-blind study, however, improvement was noted (Leiberman and Guglielmelli, 1964).

Sullivan et al. (1968a), in a preliminary report, found that dipyridamole in a dose of $400 \mathrm{mg}$. daily significantly reduced the incidence of emboli in patients who had undergone prosthetic cardiac valve replacement.

The modification of platelet aggregation by dipyridamole prompted us to examine the possibility that dipyridamole could influence the natural history of cerebral vascular disease. The daily dosage used in the cardiological studies has been of the

\footnotetext{
* Research Assistant.

† Neurological Registrar.

$¥$ Consultant Neurologist.

North Staffordshire Royal Infirmary, Stoke on Trent, Staffs.
}

order of $100 \mathrm{mg}$. Since this appears to be an ineffective dose we used a dose of $400 \mathrm{mg}$. daily in the first instance, and when no beneficial effect at this dose level was apparent we decided to double the dose.

\section{Method of Study}

A total of 169 patients entered the trial. Each had partially or completely recovered from a clinical episode of cerebral ischaemia. In setting up the trial consideration was given to the known variations in the behaviour of cerebral vascular disease. It was therefore decided to match patients in pairs according to the following criteria: (1) sex; (2) age (within five years) ; (3) presence of hypertension (diastolic blood pressure of $100 \mathrm{~mm}$. Hg or more) ; (4) clinical diagnosis at presentation according to the following subgroups: (a) transient ischaemic attacks (episodes of cerebral ischaemia lasting less than one hour), (b) single stroke, and (c) multiple strokes; and (5) length of history before beginning treatment.

Before treatment all patients underwent full clinical assessment, a full blood count was made, and an electrocardiogram taken. Liver and renal function tests were done before and six months after the beginning of treatment.

After the pairing, randomization was carried out by the statistical department of Boehringer Ingelheim Ltd., who supplied both the drug and the placebo. The active tablets contained $100 \mathrm{mg}$. of dipyridamole. The placebo tablets were of identical appearance. The information on the drug used in each patient was available in sealed envelopes throughout the trial. The patients were seen at monthly intervals for three months and then at three-monthly intervals by two observers. The clinical state was noted on each visit and any ischaemic episodes were recorded. Supplies of the drug were renewed at the time.

\section{Results and Conclusions}

Eighty-five patients received dipyridamole and 84 placebo. The relevant data pertaining to the two groups are set out in Table I. It will be seen that the treated and control groups are comparable with respect to the factors listed. Since the two groups were homogeneous, pairing was ignored at the final evaluation. The duration of disease before treatment ranged from three months to five years in both groups: $66 \%$ of the treated and $64 \%$ of the control group had a history of less than 12 months. 
On entering the trial patients were asked to take one tablet four times daily, so that those on the drug took $400 \mathrm{mg}$. of dipyridamole daily. The results of this course of treatment were assessed after an average period of observation of 14 months (first evaluation, Table II). The duration of this part of the study ranged from 6 to 24 months, and patients on treatment for less than six months were excluded. At the first

TABLE I

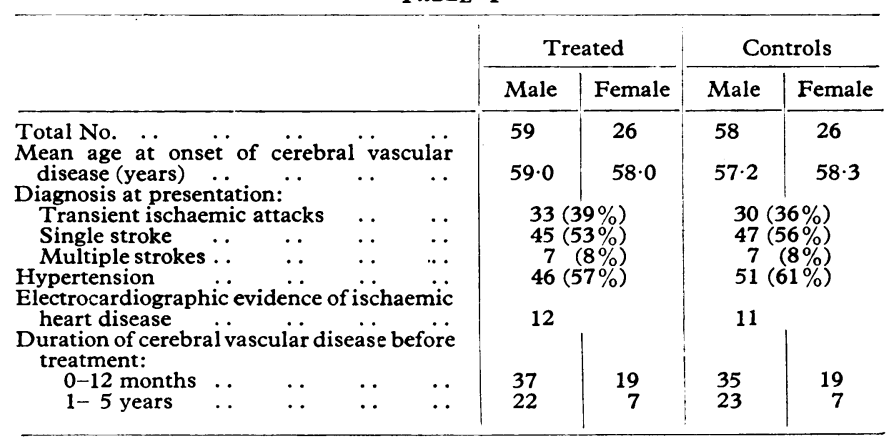

evaluation it was found that 14 had died (eight treated and six controls), and 16 were eliminated because of failure to take tablets (eight treated and eight controls). This left 69 patients in the treated and 70 patients in the control group suitable for analysis.

The dose of tablets was then doubled and the trial continued for a mean period of 11 months, when the results were again assessed (second evaluation, Table II). No patient was included who had taken the higher dose for less than nine months, and the range of the duration of treatment was 9 to 13 months. This time 57 patients in the treated and 63 patients in the control group were available for analysis. Eight further deaths had occurred (five treated and three controls), and 11 were eliminated for failure to take tablets (seven treated and four controls).

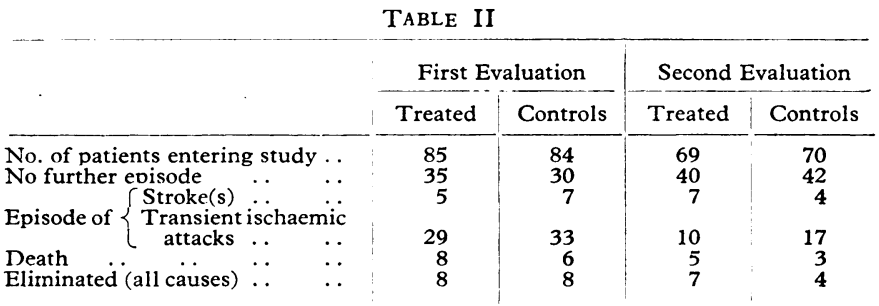

Patients eliminated from the trial because of failure to take tablets included those who discontinued because of side-effects, those who refused to complete the trial, and those who were unsuitable for analysis because the duration of treatment was too brief. The major side-effect of dipyridamole was headache, which was noted in $36(42 \%)$ of the treated group. This was of the typical vascular type, and usually cleared within 14 days. Two patients discontinued the drug because of diarrhoea, one only after the dose was raised from 400 to $800 \mathrm{mg}$. daily. No abnormality was noted in the repeat liver and renal function tests.

Twenty-two patients died during the course of the trial, 13 from the treated and nine from the control group. These patients were classed with those eliminated for other reasons. The cause of death was "cerebral vascular accident" in seven (four treated and three controls), coronary artery disease in 13 (seven treated and six controls), barbiturate poisoning in one treated patient, and bronchopneumonia in one treated patient.
Statistical analysis of the results of treatment in each of the three diagnostic categories showed no significant difference at the $5 \%$ level in the incidence of further ischaemic episodes in patients presenting with a stroke or with transient ischaemic attacks. The small number of patients presenting with multiple strokes precluded any useful comparison.

In the group presenting with transient cerebral ischaemic attacks the controls fared as well as the treated patients. Attacks continued in both groups, and in three of the treated group a further stroke occurred, as compared with two in the control group. Death occurred in four of the treated group and in one of the controls.

In patients presenting with a history of a single stroke, 24 of the treated group and 18 of the control group remained free of any further episode of cerebral ischaemia at the first evaluation. At this time 18 of the controls had noted episodes of transient ischaemia, whereas these occurred in only 11 of the treated group. The difference, however, was not significant $(\mathbf{P}=0 \cdot 1)$. At the second evaluation the number of patients free of ischaemic episodes was about equal in the two groups. Again more patients in the control (10) than in the treated group (3) reported transient ischaemic attacks. This difference failed to reach statistical significance.

When the fate of patients was followed from the lower-dose to the higher-dose stage of treatment, it became apparent that those who had episodes during the lower-dose stage tended to continue with episodes during the second stage, whereas those who were free from ischaemic episodes during the first stage tended to remain free of them during the second stage as well. Of 60 patients who had no episode during the first stage, only $10(17 \%)$ had episodes later, whereas of 69 patients who had episodes during the first stage, $44(63 \%)$ also had episodes during the second stage.

Our conclusion, therefore, is that dipyridamole in a dose of either 400 or $800 \mathrm{mg}$. daily did not appear to affect the natural history of cerebral vascular disease over the period of time covered by this study.

We are indebted to Boehringer Ingelheim Ltd. for the supply of the dipyridamole and placebo, and to Dr. M. Gent, department of statistics, Bradford University, for the statistical analysis of the results.

One of us (J. A.) is in receipt of a grant from the British Heart Foundation.

\section{REFERENCES}

Afonso, S., and O'Brien, G. S. (1967). Circulat. Res., 20, 403. Asada, S., Chiba, T., Osawa, K., Nakamura, K., and Murakawa, S. (1962). Fap. Circulat. F. (En.), 26, 849.

Chiba, T. (1963). Bull. Osaka med. Sch., 9, 106.

DeGraff, A. C., and Lyon, A. F. (1963). Amer. Heart F., 65, 423

Emmons, P. R., Harnison, M. J. G., Honour, A. J., and Mitchell, J. R. A. (1965a). Lancet, 2, 603.

Emmons, P. R., Harrison, M. J. G., Honour, A. J., and Mitchell, J. R. A. (1965b). Nature (Lond.), 208, 255.

Fam, W. M., Ragheb, S., and Hoeschen, R. J. (1964). Canad. med. Ass.

Fischer, F. 970. 217.

Foulds, T., and Mackinnon, J. (1960). Brit. med. F., 2, 835.

Laudahn, G. (1961). Experientia (Basel), 17, 415.

Leiberman, A., and Guglielmelli, S. (1964). Angiology, 15, 290

Meesmann, W., and Bachmann, G. W. (1966). Arzneimittel-Forsch., 16, 501 .

Newhouse, M. T., and McGregor, M. (1965). Amer. F. Cardiol., 16, 234

Sbar, S., and Schlant, R. C. (1967). f. Amer. med. Ass., 201, 865.

Siess, M. (1962). Arzneimittel-Forsch., 12, 683.

Soloff, L. A., Gimenez, J. L., and Winters, W. L. (1962). Amer. f. med. Sci., 243, 783 .

Sullivan, J. M., Harken, D. E., and Gorlin, R. (1968a). New Engl. f. Med., 279, 576.

Sullivan,'J. M., Kagnoff, M. F., and Gorlin, R. (1968b). Amer. f. med. Sci., 255, 292.

Vineberg, A. M., Chari, R. S., Pifarre, R., and Mercier, C. (1962). Canad. med. Ass. F., 87, 336.'

Zion, M. M., and Bradlow, B. A. (1961). S. Afr. med. F., 35, 11. 